\title{
Risk of Severe Adverse Maternal and Neonatal Outcomes in Deliveries with Repeated and Primary Cesarean Deliveries versus Vaginal Deliveries: A Cross-Sectional Study
}

\author{
Kiattisak Kongwattanakul $\mathbb{D}$, Rungroj Thamprayoch $(\mathbb{D}$, Chumnan Kietpeerakool $\mathbb{D}$, \\ and Pisake Lumbiganon
}

Department of Obstetrics and Gynecology, Faculty of Medicine, Khon Kaen University, 40002, Thailand

Correspondence should be addressed to Kiattisak Kongwattanakul; kiattisak@kku.ac.th

Received 13 July 2019; Revised 6 February 2020; Accepted 22 April 2020; Published 4 May 2020

Academic Editor: Olav Lapaire

Copyright (c) 2020 Kiattisak Kongwattanakul et al. This is an open access article distributed under the Creative Commons Attribution License, which permits unrestricted use, distribution, and reproduction in any medium, provided the original work is properly cited.

\begin{abstract}
Objective. To determine risks of severe adverse maternal and neonatal outcomes in women with repeated cesarean delivery (CD) and primary CD compared with those with vaginal delivery (VD). Methods. Data of this cross-sectional study were extracted from 2,262 pregnant women who gave birth between August 2014 and December 2016, at Srinagarind Hospital, Khon Kaen University. Severe maternal outcomes were categorized based on the World Health Organization criteria. Adjusted odds ratio (aOR) and 95\% confidence intervals (CI) were calculated to indicate the risk of severe adverse maternal and neonatal outcomes among women underwent CD compared with those who underwent VD. Results. There were no cases of maternal death in this study. CD significantly increased risk of severe adverse maternal outcomes (SMO) (aOR 10.59; 95\% CI, 1.19-94.54 for primary $\mathrm{CD}$ and aOR $17.21 ; 95 \% \mathrm{CI}, 1.97-150.51$ for repeated CD) compared with women who delivered vaginally. When compared with vaginal delivery, the risks of neonatal near miss (NNM) and severe adverse neonatal outcomes (SNO) were significantly higher in primary CD group (aOR 1.71; 95\% CI 1.17-2.51 and aOR 1.66; 95\% CI 1.14-2.43), respectively. For repeated CD, the risks were borderline significant (aOR, 1.58; 95\% CI, 0.98-2.56 for NNM and aOR, 1.61; 95\% CI, 0.99-2.60 for SNO). Conclusion. Primary and repeated CD significantly increased the risk of SMO compared with VD. Risks of NNM and SNO were also significantly increased in women with primary CD. The risks of NNM and SNO for repeated CD trended toward a significant increase.
\end{abstract}

\section{Introduction}

Cesarean delivery $(\mathrm{CD})$ is a common obstetric procedure that aims to decrease severe adverse maternal and neonatal outcomes. In 2015, the World Health Organization (WHO) however stated that "caesarean section rates higher than $10 \%$ are not associated with reductions in maternal and newborn mortality rates" [1]. In recent years, cesarean delivery has become increasingly common in both developed and developing countries. The estimated global rate of cesarean delivery between 1990 and 2014 was approximately $18.6 \%$ [2]. The absolute increase in Asia was $15.1 \%$. In Thailand, the cesarean delivery rate was $34.1 \%$ during 2007-2008 [3].
However, there is no clear evidence that the rapid increase in the number of cesarean deliveries has led to improvements in the rates of maternal or neonatal morbidity [4]. Conversely, the rates of adverse maternal and neonatal outcomes have increased significantly in the last decade [5-7]. Women with repeated cesarean deliveries also have a greater risk of placenta abnormalities [8], such as placenta previa, [9] placental abruption, placenta accreta, uterine rupture, and unplanned hysterectomy [10] in subsequent pregnancies compared with women whose previous deliveries were vaginal. Additionally, the risk of various serious maternal morbidities has increased progressively as the number of women who have undergone cesarean deliveries has grown [11]. However, some studies found no statistically significant 
differences in terms of maternal morbidities, such as placenta previa and blood transfusion, between pregnant women who have had a single repeated cesarean deliveries and those who have undergone two or more [12].

In 2011, the WHO defined the term "maternal nearmiss" (MNM) as "a woman who almost dies but survives a complication during pregnancy, childbirth, or within 42 days after termination of pregnancy" and suggested that MNM events and maternal deaths should be coupled to reflect severe maternal outcomes (SMO) [13]. The WHO MNM criteria help to identify issues that may lead to life-threatening conditions and can be used to monitor and improve the quality of care in maternity settings. This guide has been increasingly used as a tool to evaluate and improve the quality of maternal healthcare in many countries.

There have only been two previous studies that have explored cesarean deliveries in relation to MNM and SMO in low- and middle-income countries $[14,15]$, both of which indicated that women who had undergone repeated cesarean deliveries had an increased risk of MNM and SMO. However, a study from Tanzania [16] found that a history of cesarean delivery did not increase the risk of severe adverse maternal outcomes and, in fact, reduced the risk of severe adverse perinatal outcomes. Additionally, differences in terms of standards of healthcare, clinical decision making, number of women at risk, etc. may have influenced these outcomes. There has been limited data published about the risk of MNM and SMO among women with repeated CD in Thailand. The purpose of our study was to explore the risks of severe adverse maternal and neonatal outcomes in women with repeated $\mathrm{CD}$ and primary $\mathrm{CD}$ compared with those with vaginal deliveries (VD) using WHO MNM criteria.

\section{Materials and Methods}

A cross-sectional study was performed based on the medical records of pregnant women who gave birth between August 2014 and December 2016, at Khon Kaen University Faculty of Medicine's Srinagarind Hospital in Thailand. Study approval was obtained from The Khon Kaen University Ethics Committee for Human Research (HE601136).

2.1. Population and Sampling. Women who delivered with repeated cesarean section between August 2014 and December 2016, at Khon Kaen University Faculty of Medicine's Srinagarind Hospital in Thailand, were identified from labor room log books and were classified as first exposure group. One woman with primary $\mathrm{CD}$ was matched with each woman in the first exposure group by the closest date of delivery served as the second exposure group. One woman with VD was matched with each woman in the first exposure group by the closest date of delivery served as the control group.

Exclusion criteria were gestational age $<22$ weeks or birth weight $<500 \mathrm{~g}$, multifetal pregnancies, pregnancies being terminated due to fetal structural anomalies or chromosome abnormalities, and incomplete or missing data.

Medical records of these three groups of patients as well as their newborns were reviewed. Relevant data were extracted from these medical records and recorded in the pretested data collection forms.

2.2. Sample Size. The sample size was calculated for hypothesis testing of two independent proportions using the type $1(\alpha)$ error of 0.05 and power $(1-\beta)$ of 0.8 . A study by Lumbiganon et al. found the rate of severe maternal outcomes occurring in vaginal deliveries to be $1.6 \%$ [3]. We postulated that the rate of severe maternal outcomes in women with repeated CD would be 2.5 times higher. Calculations according to these values indicated a minimal sample size of 741 in each group.

2.3. Variables and Definitions. The primary outcomes were categorized into maternal and neonatal outcomes based on WHO MNM criteria [13]. Severe adverse maternal outcomes (SMO) were maternal death (MD) and maternal near-miss (MNM) which was morbidity with $>1$ organ dysfunction/life-threatening condition occurring within seven days of delivery. These included (1) cardiovascular dysfunction (defined as shock or cardiac arrest [absence of pulse/heart beat and loss of consciousness]), use of continuous vasoactive drugs, cardiopulmonary resuscitation, severe hypoperfusion (lactate $>5 \mathrm{mmol} / \mathrm{l}$ or $>45 \mathrm{mg} / \mathrm{dl})$, severe acidosis $(\mathrm{pH}<7.1)$; (2) respiratory dysfunction was defined as acute cyanosis, gasping, severe tachypnea (respiratory rate $>40$ breaths per minute), severe bradypnea (respiratory rate $<6$ breaths per minute), intubation and ventilation not related to anaesthesia, severe hypoxemia (O2 saturation $<90 \%$ for $\geq 60$ minutes or PAO2/ FiO2 <200); (3) renal dysfunction was defined as oliguria non-responsive to fluids or diuretics, dialysis for acute renal failure, severe acute azotemia (creatinine $\geq 300 \mu \mathrm{mol} / \mathrm{ml}$ or $\geq 3.5 \mathrm{mg} / \mathrm{dl}$ ); (4) coagulation/hematological dysfunction was defined as failure to form clots, massive transfusion of blood or red cells ( $\geq 5$ units), severe acute thrombocytopenia ( $<50,000$ platelets/ml); (5) hepatic dysfunction was defined as jaundice in the presence of preeclampsia, severe acute hyperbilirubinemia (bilirubin $>100 \mu \mathrm{mol} / \mathrm{l}$ or $>6.0 \mathrm{mg} / \mathrm{dl}$ ); (6) neurological dysfunction was defined as prolonged unconsciousness (lasting $\geq 12$ hours)/coma (including metabolic coma), stroke, uncontrollable fits/status epilepticus, total paralysis; and (7) uterine dysfunction was defined as uterine haemorrhage or infection leading to hysterectomy.

Neonatal near miss (NNM) was defined as Apgar score at $5 \mathrm{~min}$ of $<7$, or neonatal resuscitation, or admission to the neonatal intensive care unit occurring within seven days of delivery. Severe adverse neonatal outcomes (SNO) were a combination of neonatal death and neonatal near miss (NNM).

Potential confounding factors were fetal birth weight, maternal characteristics, and medical/obstetrical conditions. Maternal characteristics included age, level of education, pregestational BMI, and parity. Medical conditions were defined as prepregnancy diabetes mellitus, chronic hypertension, hepatitis B carrier, HIV, heart diseases, renal diseases, thyroid diseases, respiratory disease, systemic lupus erythematosus, and anemia. Obstetrical conditions were defined as pregnancy-induced hypertension, gestational diabetes mellitus. 


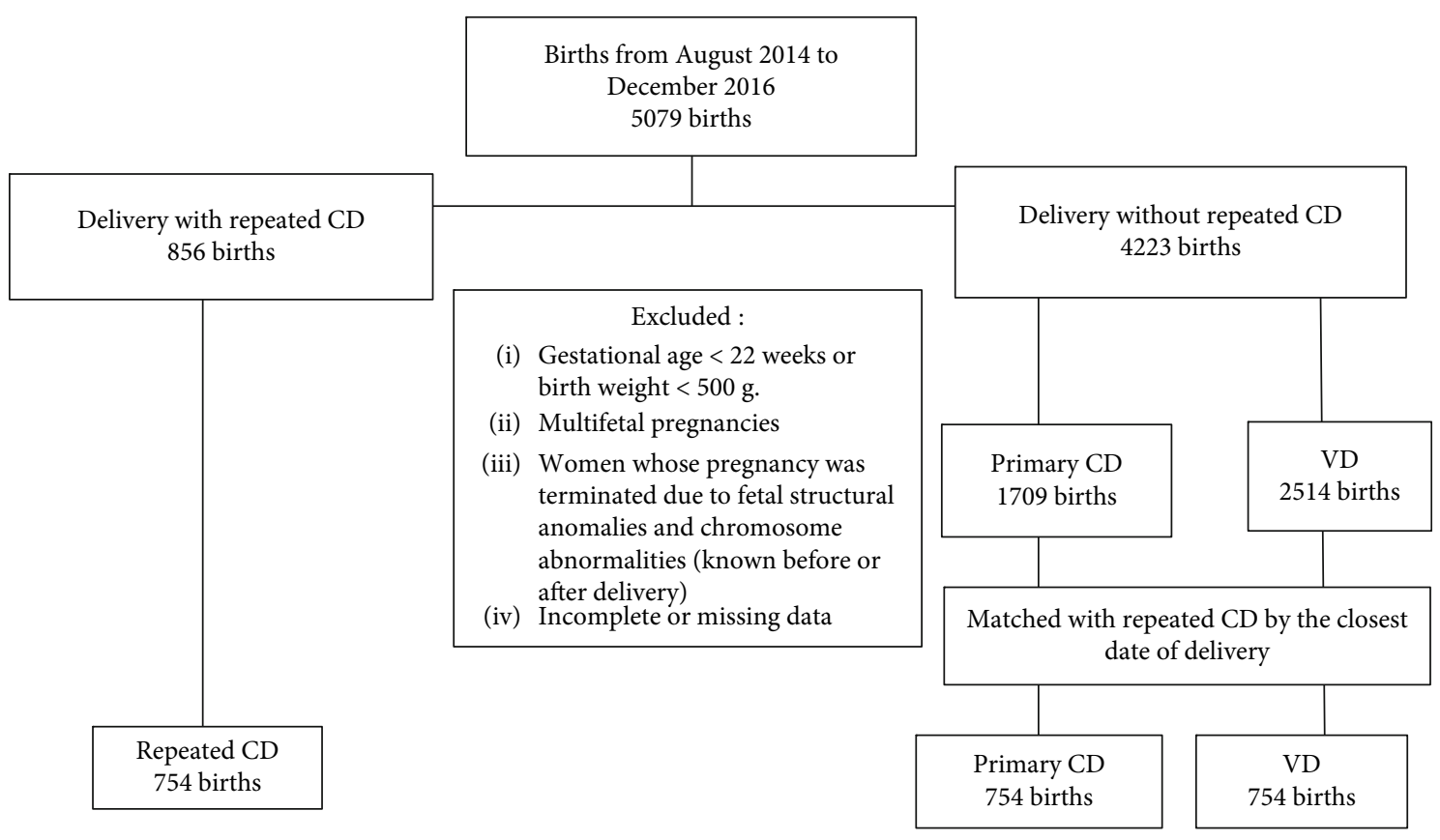

Figure 1: Flow diagram of participants.

2.4. Statistical Analysis. Descriptive statistics for gestational age at delivery (weeks), pregestational BMI, level of education, parity, and comorbidities during the current pregnancy were expressed as percentages. Multiple logistic regression was used to control potential confounding factors as described above. Risks of severe adverse maternal and neonatal outcomes among women with repeated $\mathrm{CD}$ and primary $\mathrm{CD}$ were compared with those with VD and were presented as adjusted odds ratio (aOR) with corresponding 95\% confidence intervals (CI). All analyses were carried out using STATA 10 (Stata Corporation, College Station, TX, USA).

\section{Results}

Figure 1 shows the study profile. There were 856 women delivered by repeated CD between August 2014 and December 2016. We excluded 102 women with exclusion criteria, leaving 754 women with repeated CD in the study. We systematically selected 754 women with primary CD and 754 women with vaginal deliveries matched by the closet dates of delivery.

The characteristics of the study participants are presented descriptively in Table 1. Vaginal deliveries had the highest numbers of maternal age below 20 years. Bachelor's degree or higher was the most frequent level of education of women in the present study. Underweight pregestational BMI was lower in women with repeated CD, whereas overweight/obesity was higher in this group. Multiparous was the lowest in women with primary $\mathrm{CD}$. The most common medical condition during pregnancy was anemia.

Table 2 shows severe adverse maternal and neonatal outcomes by mode of delivery. There were no maternal deaths. Hematological dysfunction and uterine dysfunction were the two most common MNM outcomes. Severe postpartum haemorrhage was higher in women with cesarean deliveries. There was no placenta accrete syndromes and admission to ICU in women with VD, whereas sepsis or severe systemic infection was higher in women with VD. There were five neonatal deaths; one $(0.13 \%)$ in women with primary $\mathrm{CD}$ group, one $(0.13 \%)$ in women with repeated $\mathrm{CD}$, and three $(0.40 \%)$ in women with VD.

Table 3 shows maternal outcomes by mode of delivery. The odds of SMO was significantly increased in women with primary CD (adjusted OR 10.59, 95\% CI 1.19-94.54) and in women with repeated CD (adjusted OR 17.21, 95\% CI 1.97-150.51) compared with women with VD. Severe postpartum haemorrhage was significantly increased in women with both primary and repeated CD compared with women with VD (adjusted OR 12.73, 95\% CI 1.53-105.55 and $15.93,95 \%$ CI 1.83-138.29, respectively).

Table 4 shows severe adverse neonatal outcomes (SNO) by mode of delivery. The odds of SNO was significantly increased in women with primary $\mathrm{CD}$ compared to women with VD (adjusted OR 1.66, 95\% CI 1.14-2.43). The odds of SNO were also increased in women who with repeated CD compared with VD. However, this difference was not statistically significant, although borderline (adjusted OR 1.61 95\% CI 0.99-2.60).

\section{Discussion}

Women undergoing $\mathrm{CD}$ carried significantly higher risks of MNM, SMO, and severe postpartum haemorrhage. For neonatal outcomes, neonates born by $\mathrm{CD}$ had higher rates of NNM and SNO compared with those who were born vaginally. When adjusted by potential confounding factors including maternal age, parity, birth weight, and maternal comorbidity, the higher risks of these adverse pregnancy 
TABLE 1: Baseline characteristics of women with vaginal, primary and repeated cesarean deliveries.

\begin{tabular}{|c|c|c|c|}
\hline Baseline characteristics & $\begin{array}{c}\mathrm{VD}(n=754) \\
n(\%)\end{array}$ & $\begin{array}{c}\text { Primary CD }(n=754) \\
n(\%)\end{array}$ & $\begin{array}{c}\text { Repeated CD }(n=754) \\
n(\%)\end{array}$ \\
\hline \multicolumn{4}{|l|}{ Maternal characteristics } \\
\hline \multicolumn{4}{|l|}{ Age (year) } \\
\hline$<20$ & $61(8.09)$ & $27(3.58)$ & $2(0.27)$ \\
\hline $20-34$ & $623(82.63)$ & $551(73.08)$ & $536(71.09)$ \\
\hline$>35$ & $70(9.28)$ & $176(23.34)$ & $216(28.65)$ \\
\hline \multicolumn{4}{|l|}{ Level of education } \\
\hline$<$ high school & $87(11.66)$ & $74(9.87)$ & $69(9.25)$ \\
\hline High school & $189(25.34)$ & $111(14.80)$ & $74(9.92)$ \\
\hline Vocational school & $156(20.91)$ & $133(17.73)$ & $136(18.23)$ \\
\hline Bachelor's degree or higher & $314(42.09)$ & $432(57.60)$ & $467(62.60)$ \\
\hline \multicolumn{4}{|l|}{ Pregestational BMI } \\
\hline$<18.5$ (underweight) & $212(28.30)$ & $153(20.48)$ & $102(13.60)$ \\
\hline $18.5-24.9$ (normal) & $464(61.95)$ & $467(62.52)$ & $462(61.60)$ \\
\hline 25-29.9 (overweight) & $48(6.41)$ & $91(12.18)$ & $134(17.87)$ \\
\hline$>30$ (obese) & $25(3.34)$ & $36(4.82)$ & $52(6.93)$ \\
\hline Multiparous & $329(43.63)$ & $149(19.76)$ & $754(100)$ \\
\hline \multicolumn{4}{|l|}{ Medical condition } \\
\hline Prepregnancy diabetes mellitus & $1(0.13)$ & $2(0.27)$ & $1(0.13)$ \\
\hline Chronic hypertension & $3(0.40)$ & $4(0.53)$ & $14(1.86)$ \\
\hline Hepatitis B carrier & $15(1.99)$ & $22(2.92)$ & $19(2.52)$ \\
\hline HIV & $2(0.27)$ & $3(0.40)$ & $4(0.53)$ \\
\hline Heart diseases & $3(0.40)$ & $6(0.80)$ & $5(0.66)$ \\
\hline Renal diseases & $2(0.27)$ & $3(0.40)$ & $3(0.40)$ \\
\hline Thyroid diseases & $11(1.46)$ & $14(1.86)$ & $7(0.93)$ \\
\hline Respiratory disease & $3(0.40)$ & $4(0.53)$ & $3(0.40)$ \\
\hline Systemic lupus erythematosus & $2(0.27)$ & $8(1.06)$ & $3(0.40)$ \\
\hline Anemia & $113(14.99)$ & $126(16.71)$ & $120(15.92)$ \\
\hline \multicolumn{4}{|l|}{ Obstetric condition } \\
\hline Pregnancy induced hypertension & $19(2.52)$ & $41(5.44)$ & $22(2.92)$ \\
\hline Gestational diabetes mellitus & $38(5.04)$ & $76(10.08)$ & $72(9.55)$ \\
\hline \multicolumn{4}{|l|}{ Neonatal characteristics } \\
\hline \multicolumn{4}{|l|}{ Birthweight (g) } \\
\hline$<2,500$ & $69(9.16)$ & $71(9.43)$ & $57(7.56)$ \\
\hline $2,500-4,000$ & $677(89.91)$ & $654(86.85)$ & $676(89.66)$ \\
\hline$>4,000$ & $7(0.93)$ & $28(3.72)$ & $21(2.79)$ \\
\hline \multicolumn{4}{|l|}{ Gestational age at delivery (week) } \\
\hline Preterm $(<37)$ & $70(9.28)$ & $63(8.36)$ & $86(11.41)$ \\
\hline Term $(37-41)$ & $683(90.58)$ & $690(91.51)$ & 668 (88.59) \\
\hline Postterm $(>42)$ & $1(0.13)$ & $1(0.13)$ & $0(0.00)$ \\
\hline
\end{tabular}

VD: vaginal delivery; CD: cesarean delivery.

outcomes appeared to be in the same direction for both primary and repeated CD groups.

MNM and SMO have been acknowledged as the outcomes that are strongly associated with $\mathrm{CD}[17,18]$. Mohammadi et al. [17] reported that the most significant predictor was the route of delivery. Women undergoing CD were approximately a 7.4-fold likelihood of experiencing MNM (95\% CI, 3.7-15.1). A study conducted in Northern
Ethiopia reported that $\mathrm{CD}$ has increased the odds of experiencing MNM (adjusted OR 4.6; 95\% CI: 1.98, 7.61). [18] In a population-based cohort study that covered 900,108 women aged 15-44 years with singleton live births, $\mathrm{CD}$ was associated with a significantly increased risk of postpartum readmission (rate 2.7\%; OR, 1.9; 95\% CI, 1.8-1.9) compared with vaginal delivery [19]. The common postpartum complications consisted of pelvic injury/wound 
TABLE 2: Maternal and neonatal outcomes of women with vaginal, primary and repeated cesarean deliveries.

\begin{tabular}{|c|c|c|c|}
\hline & $\begin{array}{c}\mathrm{VD}(n=754) \\
n(\%)\end{array}$ & $\begin{array}{c}\text { Primary CD }(n=754) \\
n(\%)\end{array}$ & $\begin{array}{c}\text { Repeated CD }(n=754) \\
n(\%)\end{array}$ \\
\hline \multicolumn{4}{|l|}{ Maternal outcome } \\
\hline \multicolumn{4}{|l|}{ Maternal vital status } \\
\hline (i) Maternal death & $0(0.00)$ & $0(0.00)$ & $0(0.00)$ \\
\hline \multicolumn{4}{|l|}{ Severe maternal complications } \\
\hline (i) Severe postpartum haemorrhage & $1(0.13)$ & $10(1.33)$ & $16(2.12)$ \\
\hline (ii) Sepsis or severe systemic infection & $13(1.72)$ & $4(0.53)$ & $3(0.40)$ \\
\hline (iii) Placental previa & $0(0.00)$ & $20(2.65)$ & $21(2.79)$ \\
\hline (iv) Placenta accrete syndromes & $0(0.00)$ & $2(0.27)$ & $8(1.06)$ \\
\hline (v) Placenta abruption & $1(0.13)$ & $4(0.53)$ & $0(0.00)$ \\
\hline (vi) Ruptured uterus & $0(0.00)$ & $0(0.00)$ & $0(0.00)$ \\
\hline \multicolumn{4}{|l|}{ Critical interventions } \\
\hline (i) Admission to intensive care unit & $0(0.00)$ & $12(1.59)$ & $16(2.12)$ \\
\hline (ii) Interventional radiology & $0(0.00)$ & $0(0.00)$ & $0(0.00)$ \\
\hline (iii) Laparotomy (includes hysterectomy, excludes caesarean section) & $0(0.00)$ & $2(0.27)$ & $4(0.53)$ \\
\hline (v) Massive blood transfusion & $0(0.00)$ & $4(0.53)$ & $8(1.06)$ \\
\hline \multicolumn{4}{|l|}{ Maternal near-miss } \\
\hline (i) Cardiovascular dysfunction & $0(0.00)$ & $2(0.27)$ & $1(0.13)$ \\
\hline (ii) Respiratory dysfunction & $0(0.00)$ & $5(0.66)$ & $7(0.93)$ \\
\hline (iii) Renal dysfunction & $0(0.00)$ & $0(0.00)$ & $2(0.27)$ \\
\hline (iv) Hepatic dysfunction & $0(0.00)$ & $0(0.00)$ & $0(0.00)$ \\
\hline (v) Neurological dysfunction & $0(0.00)$ & $1(0.13)$ & $0(0.00)$ \\
\hline (vi) Hematological dysfunction & $1(0.13)$ & $4(0.53)$ & $8(1.06)$ \\
\hline (vii) Uterine dysfunction & $0(0.00)$ & $2(0.27)$ & $10(1.33)$ \\
\hline \multicolumn{4}{|l|}{ Neonatal outcomes } \\
\hline (i) Apgar score at 5 minutes $<7$ & $5(0.66)$ & $14(1.86)$ & $11(1.46)$ \\
\hline (ii) Neonatal resuscitation & $59(7.82)$ & $99(13.13)$ & $69(9.15)$ \\
\hline (iii) NICU admission & $15(1.99)$ & $30(3.98)$ & $23(3.05)$ \\
\hline (iv) Intrapartum deaths & $3(0.40)$ & $0(0.00)$ & $1(0.13)$ \\
\hline (v) Early neonatal death & $0(0.00)$ & $1(0.13)$ & $0(0.00)$ \\
\hline
\end{tabular}

CD: cesarean delivery; VD: vaginal delivery.

TABLe 3: Maternal outcomes by mode of delivery.

\begin{tabular}{lccccc}
\hline \multirow{2}{*}{ Maternal outcome } & VD & \multicolumn{2}{c}{ Primary CD } & \multicolumn{2}{c}{ Repeated CD } \\
& Reference group & Crude OR [95\% CI] & aOR [95\% CI] & Crude OR [95\% CI] & aOR [95\% CI] \\
\hline Maternal death & 1 & - & - & - & - \\
Maternal near miss (MNM) & 1 & $9.10(1.15-71.98)$ & $10.59(1.19-94.54)$ & $16.32(2.16-123.41)$ & $17.21(1.97-150.51)$ \\
Severe maternal outcomes (SMO) & 1 & $9.10(1.15-71.98)$ & $10.59(1.19-94.54)$ & $16.32(2.16-123.41)$ & $17.21(1.97-150.51)$ \\
Severe postpartum haemorrhage & 1 & $10.12(1.29-79.26)$ & $12.73(1.53-105.55)$ & $16.33(2.16-123.41)$ & $15.93(1.83-138.29)$ \\
\hline
\end{tabular}

Adjusted by maternal age, level of education, parity, pregestational BMI, medical and obstetrical conditions, and birth weight (g). Women with vaginal deliveries are the reference. $\mathrm{CD}$ : cesarean delivery; VD: vaginal delivery.

complication, obstetric complications, venous disorders and thromboembolism, and major puerperal infection [19]. Our findings confirm that $\mathrm{CD}$ is independently associated with MNM and SMO regardless of the type of CD.

Although there were no maternal deaths in this study, a recent systematic review conducted to assess the relationship between maternal death and CD in Latin America indicated an increased risk of maternal death following CD compared to vaginal delivery [20]. An elevated risk of maternal death among women undergoing $\mathrm{CD}$ is also noted in highincome countries. In the Netherlands, the risk of death after CD was 21.9 per $100.000 \mathrm{CD}$ performed compared to that of 3.8 deaths per 100.000 vaginal births. Compared to vaginal birth, maternal mortality after $\mathrm{CD}$ was 3.4 times higher 
TABLE 4: Neonatal outcomes by mode of delivery.

\begin{tabular}{lccccc}
\hline \multirow{2}{*}{ Neonatal outcomes } & VD & \multicolumn{2}{c}{ Primary CD } & \multicolumn{2}{c}{ Repeated CD } \\
& Reference group & Crude OR [95\% CI] & aOR [95\% CI] & Crude OR [95\% CI] & aOR [95\% CI] \\
\hline Neonatal deaths & 1 & $0.33(0.03-3.20)$ & $0.34(0.01-6.73)$ & $0.33(0.03-3.20)$ & Not estimable \\
Neonatal near miss (NNM) & 1 & $1.80(1.28-2.53)$ & $1.71(1.17-2.51)$ & $1.21(0.84-1.73)$ & $1.58(0.98-2.56)$ \\
Severe neonatal outcomes (SNO) & 1 & $1.73(1.24-2.41)$ & $1.66(1.14-2.43)$ & $1.16(0.81-1.66)$ & $1.61(0.99-2.60)$ \\
Apgar score at 5 minutes $<7$ & 1 & $2.83(1.02-7.91)$ & $3.74(1.06-13.17)$ & $2.21(0.77-6.41)$ & $7.45(1.28-43.46)$ \\
Neonatal resuscitation & 1 & $1.78(1.27-2.50)$ & $1.72(1.17-2.53)$ & $1.19(0.83-1.71)$ & $1.56(0.96-2.52)$ \\
Preterm birth $(<37$ weeks) & 1 & $0.89(0.62-1.27)$ & $0.88(0.55-1.41)$ & $1.26(0.90-1.76)$ & $1.09(0.68-1.74)$ \\
Low birth weight $(<2500 \mathrm{~g})$ & 1 & $1.03(0.73-1.46)$ & $0.90(0.60-1.35)$ & $0.81(0.56-1.17)$ & $0.93(0.58-1.49)$ \\
\hline
\end{tabular}

Adjusted by maternal age, level of education, parity, pregestational BMI, medical and obstetrical conditions and birth weight (g). Women with vaginal delivery is the reference. $\mathrm{CD}$ : cesarean delivery; VD: vaginal delivery.

(95\% CI, 2.4-4.8) after excluding deaths that had no association with surgery [21].

In many low- and middle-income countries, obstetric haemorrhage is the leading cause of serious maternal morbidity and mortality [22]. Obstetric haemorrhage accounted for approximately $26-46 \%$ of the MNM cases in Ethiopian women $[18,23]$. In this study, we determined the association between severe postpartum haemorrhage and CD. We noted that $\mathrm{CD}$ increased the risk of this maternal life-threatening condition when compared to vaginal delivery. In this investigation, we also observed that among 754 women with repeated $\mathrm{CD}$, there were eight cases (a prevalence of $1.06 \%$ ) of placenta accrete. A recent report from the US indicated that women with previous $\mathrm{CD}$ had an increased risk of placenta accrete which is now considered to be an important cause of maternal death in the US [24].

A cohort study conducted in Southeast Brazil revealed higher rates of NNM and neonatal death following CD compared with vaginal delivery. Neonates born by CD carried approximately 2 times more likely to suffer from NNM (OR, 2.0; 95\% CI, 1.35-2.96). However, the difference in terms of the risk of neonatal death among $\mathrm{CD}$ and vaginal delivery did not reach a statistically significant threshold (OR, 1.29; (95\% CI 0.61-2.74) [25]. In the present study, the rates of NNM and SNO were higher among neonates born by CD compared to those who born vaginally. The risks of these two adverse outcomes were significantly higher among those in primary $\mathrm{CD}$ group. For repeated $\mathrm{CD}$, it was found to be marginally significant when controlling for commonly applied confounding factors. However, a relatively small sample size and rarity of the occurrence of neonatal death in the present study precluded any meaningful interpretation regarding the impact of $\mathrm{CD}$ on the risk of neonatal death.

The strength of this study is that the adverse maternal and neonatal outcomes were measured as per the most recent approach recommended by WHO. Some limitations of this study however are worthy of note. First, this study applied retrospective data collection; some clinically important information may not be available, such as indications of CD, labor characteristics, adequacy of antenatal care, and smoking history. Second, a relative rarity of outcomes of interest has resulted in a wide confidence interval of summary measures. Third, the present study was conducted at a referral institution, which was likely to have an overrepresentation of complicated pregnancy. This might limit the extrapolation of our findings to facilities of primary or secondary healthcare settings.

\section{Conclusions}

The present study observed significant independently higher risks of MNM, SMO, and severe postpartum haemorrhage among women undergoing $\mathrm{CD}$ with a higher risk in repeated CD. CD has also tended to increase the risks of NNM and SNO. Based on these findings, effective interventions for reducing unnecessary $\mathrm{CD}$ are therefore of utmost importance to avoid adverse pregnancy outcomes that are potentially associated with CD.

\section{Data Availability}

The data used to support the findings of this study are available from the corresponding author upon request.

\section{Conflicts of Interest}

There is no financial or other relationship that might lead to a conflict of interest.

\section{Acknowledgments}

We thank Dylan Southard, the English Consultant, Faculty of Medicine, Khon Kaen University, for English-language presentation of this manuscript. This study was granted by the Faculty of Medicine, Khon Kaen University (grant number IN60336).

\section{References}

[1] A. P. Betran, M. R. Torloni, J. J. Zhang, A. M. Gülmezoglu, and WHO Working Group on Caesarean Section, "WHO statement on caesarean section rates," BJOG: An International Journal of Obstetrics \& Gynaecology, vol. 123, no. 5, pp. 667670, 2016.

[2] A. P. Betrán, J. Ye, A.-B. Moller, J. Zhang, A. M. Gülmezoglu, and M. R. Torloni, "The increasing trend in caesarean section rates: global, regional and national estimates: 1990-2014," PloS One, vol. 11, no. 2, article e0148343, 2016. 
[3] P. Lumbiganon, M. Laopaiboon, A. M. Gülmezoglu et al., "Method of delivery and pregnancy outcomes in Asia: the WHO global survey on maternal and perinatal health 200708," Lancet, vol. 375, no. 9713, pp. 490-499, 2010.

[4] J. Villar, E. Valladares, D. Wojdyla et al., "Caesarean delivery rates and pregnancy outcomes: the 2005 WHO global survey on maternal and perinatal health in Latin America," Lancet, vol. 367, no. 9525, pp. 1819-1829, 2006.

[5] C. Deneux-Tharaux, E. Carmona, M.-H. Bouvier-Colle, and G. Bréart, "Postpartum maternal mortality and cesarean delivery," Obstetrics and Gynecology, vol. 108, 3 Part 1, pp. 541-548, 2006.

[6] S. Liu, R. M. Liston, K. S. Joseph et al., "Maternal mortality and severe morbidity associated with low-risk planned cesarean delivery versus planned vaginal delivery at term," Canadian Medical Association Journal, vol. 176, no. 4, pp. 455-460, 2007.

[7] K. D. Gregory, S. Jackson, L. Korst, and M. Fridman, "Cesarean versus vaginal delivery: whose risks? Whose benefits?," American Journal of Perinatology, vol. 29, no. 1, pp. 7-18, 2012.

[8] R. M. Silver, M. B. Landon, D. J. Rouse et al., "Maternal morbidity associated with multiple repeat cesarean deliveries," Obstetrics and Gynecology, vol. 107, no. 6, pp. 1226-1232, 2006.

[9] I. Gurol-Urganci, D. A. Cromwell, L. C. Edozien et al., "Risk of placenta previa in second birth after first birth cesarean section: a population-based study and meta-analysis," BMC Pregnancy and Childbirth, vol. 11, no. 1, p. 95, 2011.

[10] S. C. Curtin, K. D. Gregory, L. M. Korst, and S. F. Uddin, "Maternal Morbidity for Vaginal and Cesarean Deliveries, According to Previous Cesarean History: New Data From the Birth Certificate, 2013," National vital statistics reports: from the Centers for Disease Control and Prevention, National Center for Health Statistics, National Vital Statistics System, vol. 64, no. 4, pp. 1-13, 2015.

[11] N. E. Marshall, R. Fu, and J.-M. Guise, "Impact of multiple cesarean deliveries on maternal morbidity: a systematic review," American Journal of Obstetrics and Gynecology, vol. 205, no. 3, pp. 262.e1-262.e8, 2011.

[12] P. Wuttikonsammakit and N. Sukcharoen, "Pregnancy outcomes of multiple repeated cesarean sections in King Chulalongkorn Memorial Hospital," Journal of the Medical Association of Thailand= Chotmaihet thangphaet, vol. 89, Suppl 4, pp. S81-S86, 2006.

[13] World Health Organization, Evaluating the quality of care for severe pregnancy complications: the WHO near-miss approach for maternal healthNovember 2011, http://www.who.int/ reproductivehealth/publications/monitoring/9789241502221/ en/.

[14] J. P. Souza, J. G. Cecatti, A. Faundes et al., "Maternal near miss and maternal death in the World Health Organization's 2005 global survey on maternal and perinatal health," Bulletin of the World Health Organization, vol. 88, no. 2, pp. 113-119, 2010.

[15] J. P. Souza, A. M. Gülmezoglu, J. Vogel et al., "Moving beyond essential interventions for reduction of maternal mortality (the WHO Multicountry Survey on Maternal and Newborn Health): a cross-sectional study," The Lancet, vol. 381, no. 9879, pp. 1747-1755, 2013.

[16] H. Litorp, M. Rööst, H. L. Kidanto, L. Nyström, and B. Essén, "The effects of previous cesarean deliveries on severe maternal and adverse perinatal outcomes at a university hospital in
Tanzania," International Journal of Gynecology \& Obstetrics, vol. 133, no. 2, pp. 183-187, 2016.

[17] S. Mohammadi, B. Essén, M. Fallahian, R. Taheripanah, S. Saleh Gargari, and C. Källestål, "Maternal near-miss at university hospitals with cesarean overuse: an incident casecontrol study," Acta Obstetricia et Gynecologica Scandinavica, vol. 95, no. 7, pp. 777-786, 2016.

[18] D. E. Mekango, M. Alemayehu, G. B. Gebregergs, A. A. Medhanyie, and G. Goba, "Determinants of maternal near miss among women in public hospital maternity wards in northern Ethiopia: a facility based case-control study," PloS One, vol. 12, no. 9, article e0183886, 2017.

[19] S. Liu, M. Heaman, K. S. Joseph et al., "Risk of maternal postpartum readmission associated with mode of delivery," Obstetrics and Gynecology, vol. 105, no. 4, pp. 836-842, 2005.

[20] W. M. Fahmy, C. A. Crispim, and S. Cliffe, "Association between maternal death and cesarean section in Latin America: a systematic literature review," Midwifery, vol. 59, pp. 88-93, 2018.

[21] A. F. Kallianidis, J. M. Schutte, J. van Roosmalen, and T. van den Akker, "Maternal mortality after cesarean section in the Netherlands," European Journal of Obstetrics, Gynecology, and Reproductive Biology, vol. 229, pp. 148-152, 2018.

[22] L. Say, D. Chou, A. Gemmill et al., "Global causes of maternal death: a WHO systematic analysis," The Lancet Global Health, vol. 2, no. 6, pp. e323-e333, 2014.

[23] A. W. Kasahun and W. G. Wako, "Predictors of maternal near miss among women admitted in Gurage zone hospitals, South Ethiopia, 2017: a case control study," BMC Pregnancy and Childbirth, vol. 18, no. 1, p. 260, 2018.

[24] R. M. Silver and D. W. Branch, "Placenta Accreta Spectrum," New England Journal of Medicine, vol. 378, no. 16, pp. 15291536, 2018.

[25] P. L. Kale, M. H. P. Mello-Jorgede, K. S. Silvada, and S. C. Fonseca, "Neonatal near miss and mortality: factors associated with life-threatening conditions in newborns at six public maternity hospitals in Southeast Brazil," Cadernos de Saúde Pública, vol. 33, no. 4, article e00179115, 2017. 